\title{
Diagnosis and Application of Serum Procalcitonin in Lower Respiratory Tract Infections
}

\author{
LI JUNMIN* \\ Department of Respiratory, Renmin Hospital, Hubei University of Medicine, Shiyan 442000, China
}

\author{
Junmin: Diagnosis and Application of Serum Procalcitonin
}

\begin{abstract}
The role of serum procalcitonin in early diagnosis of lower respiratory tract bacterial infections is tested and studied. The prognosis of ventilator-associated pneumonia group and nonventilator-associated pneumonia group were compared. There was no significant difference between ventilator-associated pneumonia group and nonventilator-associated pneumonia group before mechanical ventilation. After mechanical ventilation, procalcitonin, C-reactive protein and white blood cells in ventilator-associated pneumonia group were significantly higher than those in nonventilator-associated pneumonia group $(t=1.62-2.31, P<0.05)$ and the body temperature was also elevated, which is not significant. The sensitivity and specificity of procalcitonin in diagnosing ventilator-associated pneumonia were 86 and $91 \%$, respectively. Procalcitonin level in Gram negative bacteria group was significantly higher than that in Gram positive bacteria group $(\mathrm{t}=\mathbf{1 . 9 9}, \mathbf{P}<\mathbf{0 . 0 5})$. Immunofluorescence quantitative assay was used to detect respiratory infection patients and the treatment of some patients was monitored. At the same time, white blood cells and C-reactive protein were compared with procalcitonin. The sensitivity and specificity of procalcitonin in bacterial infections of respiratory system are obviously higher than those of traditional C-reactive protein and white blood cells. It can be used for early diagnosis of bacterial infections quickly. This study provided important clinical evidence in judging the severity and prognosis of infection and is helpful for rational use of antibiotics..
\end{abstract}

Key words: Diagnosis, serum procalcitonin, lower respiratory tract infections

Serum procalcitonin (PCT), as a new bacterial infection index, has been widely used in the diagnosis of infectious diseases in recent years. Lower respiratory tract infections (LRTI) are tracheal, bronchial and pulmonary infections caused by bacteria, viruses or other atypical pathogens. Because it is difficult to distinguish bacteria from viruses, it is easy to misuse antibiotics. Therefore, early identification and diagnosis of bacterial and viral infections and timely and effective treatment to reduce the growth of drug-resistant bacteria, is one of the major clinical problems. PCT, as a valuable new indicator for early identification of respiratory bacterial infections, is applied in clinic. In this study, immunofluorescence assay was used to quantitatively detect PCT in patients with respiratory diseases and the clinical significance of serum PCT in respiratory bacterial infections was discussed $^{[1-5]}$.

With the development of critical care medicine, ventilator therapy has been widely used in intensive care unit (ICU) and respiratory department, which has saved the lives of numerous critical patients. However, the accompanying ventilator-associated pneumonia (VAP) brings great difficulty and confusion to this treatment. Statistics show that the incidence of VAP is 10-20\% and the mortality rate of patients can be as high as 24$50 \%$. If early onset of the disease is not effectively treated, especially with the correct use of antibiotics, it often lead to rapid deterioration of patients, leading to death in some cases ${ }^{[6-10]}$. Many studies reported that timely and effective drug treatment after VAP can greatly reduce the treatment time and mortality of patients. All this is based on the early detection of VAP, making it very important. However, there are still many difficulties in the early diagnosis and evaluation of the severity of VAP. Many experts are looking for and improving the diagnostic methods, especially in the discovery and selection of some biological markers, in order to better solve this clinical difficulty.

Research has put forward the clinical pulmonary infection score (CPIS) system, which is composed of

*Address for correspondence

E-mail: 1913558931@qq.com 
clinical manifestations, imaging changes and laboratory etiology standards to evaluate the severity of pulmonary infection. Specific to the clinical operation is the use of patient's temperature, peripheral blood white blood cell count, respiratory tract secretion characteristics, patient's oxygenation index, chest X-ray changes and sputum pathogenic data, which were all scored and the probability of pulmonary infection was judged by the score. A simplified CPIS table was put forward on the basis of standard CPIS score to facilitate the application of this scoring system. Studies have shown that the greater the CPIS score, the higher the degree of pulmonary infection and the worse the overall prognosis of patients. If the CPIS score of patients decreased gradually during the treatment process, the patient's prognosis improved, if the value showed an increasing trend, the prognosis was poor.

The American Thoracic Association (ATS) and the Infectious Diseases Association (IDSA) point out that in hospital-acquired pneumonia (HAP), VAP guidelines that CPIS can be used to assist in the diagnosis of pulmonary infections. However, this scoring system has low sensitivity and specificity in the diagnosis of VAP. It has been reported that the area under the curve (AUC) predicted by CPIS is only 0.581. APACHE II (acute physiology and chronic health evaluation scoring system) is the most popular and authoritative system for critical patients in ICU, which can be accepted by clinicians. It is composed of acute physiology score (APS), age and chronic health status score, the sum of which is the total score ${ }^{[11-15]}$. Clinicians use this scoring system to quantify patient's condition and predict overall mortality of ICU patients and then formulate and modify the medical and nursing treatment programs so as to improve the success rate of emergency treatment of critical illnesses, in addition to providing an objective and scientific way to determine the timing of patient treatment and transfer and to rationally allocate medical resources. The basis of this, it can be used not only to compare the severity of the disease between patients with a single disease, but also to compare different diseases and patients with multiple diseases. APACHE II scoring system can be used as a reliable indicator for evaluating the severity and prognosis of ICU patients. Not only in intensive care units, but also in general wards, the APACHE II score can be used to assess critically ill patients. Studies have shown that APACHE II score has a good predictive value for patients with pulmonary infection.
C-reactive protein (CRP) is an acute positive-phase protein that reacts with C-glycosome of Diplococcus pneumoniae. It is a non-specific marker in the case of invasion of pathogenic microorganisms or other causes of tissue and organ damage. It can activate complements and promote phagocytosis during stress. Many studies have shown that CRP can be effectively used to identify bacterial and viral infections. CRP is clinically used to observe the severity of various diseases and its response to treatment. CRP in the blood of healthy people shows a low concentration, when the body encounters bacterial infection, tissue necrosis, CRP concentration quickly increased, its speed exceeds other similar indicators of inflammation monitoring, in the onset of 6 to 12 hours, serum or whole blood concentration will reach the peak concentration, with the improvement of the patient's condition, CRP value also decreased with it. Trend, the rate of decline is much faster than that of erythrocyte sedimentation rate and white blood cell count.

Leukocytes are a nuclear cell component of peripheral blood, which is significantly less than red blood cells in quantity, accounting for only 0.1-0.2\%. These are divided into granulocytes, lymphocytes and monocytes according to their morphology. Granulocytes are classified into neutrophils, eosinophils and basophils according to their staining characteristics. The white blood cell (WBC) count in the circulating peripheral blood is disturbed by many physiological reasons, such as age; the total WBC count fluctuates between $(4-10) \times 10^{9}$ in adults, from $(15-20) \times 10^{9}$ in newborns and from $(11-20)) \times 10^{9}$ in children aged 6-2. WBC count varies with time of the day, afternoon is often higher than in the morning. The difference between the highest and the lowest in a day can sometimes be doubled. WBC count is lower when the person is quiet and relaxed but it can be higher during activity such as vigorous exercise, pain, eating and emotional disturbances. WBCs increased during pregnancy and childbirth, especially in the first month before childbirth. The proportion of WBCs and types of WBCs in blood is relatively small under normal circumstances ${ }^{[16-21]}$. The proportion of neutrophils, eosinophils, basophils, lymphocytes and monocytes was 50-70, 1-4, 0-1, 20-40 and 1-7\%, respectively. When inflammation or other abnormalities occur in the body, the total number of white blood cells and the three subtypes of white blood cells can be changed. Based on the above reasons, WBC count and WBC classification count become an important method of laboratory examination. WBC detection is of great significance for clinical diagnosis. However, in 
addition to infection, there are many factors affecting WBC counts, such as immunosuppression, state of emergency and so on, so it lacks specificity towards diagnosis of infection. Usually, if the fluctuation of WBC count is not more than $30 \%$, it is not helpful for clinical diagnosis. It is meaningful only by dynamic observation.

PCT, a hormone-free procalcitonin peptide, is a glycoprotein containing 116 amino acids. It is encoded by the Calc I gene on chromosome 11 and has a relative molecular weight of $13 \mathrm{kD}$. It is a biomarker closely related to infection. Normal human serum PCT level is $<0.1 \mathrm{~g} / 1$. Normally, it is synthesized and secreted by $\mathrm{C}$ cells in the thyroid gland. When there is systemic bacterial infection, the serum PCT rises rapidly. After anti-infective treatment, the serum PCT decreases rapidly. This situation does not exist with other pathogenic microorganisms, such as viruses, fungal infections and autoimmune diseases. Some studies have reported that PCT has a certain application value in the diagnosis of VAP caused by bacterial infection. The sensitivity, specificity and diagnostic accuracy of PCT for VAP were 90.6, 84.6 and $7.9 \%$, respectively.

These values were higher than those of CRP, WBC, neutrophil ratio, body temperature sensitivity, specificity and diagnostic accuracy. $\mathrm{T}$ is superior to the above inflammatory markers in the diagnosis of VAP. Studies reported elsewhere also showed that PCT has better sensitivity and specificity than other indicators in the diagnosis of VAP. Although the sensitivity of CPIS is similar to that of PCT, the specificity is lower. However, not all clinical trials have positive results for the value of PCT in the diagnosis of VAP, and some have reported the opposite. It is important and necessary to identify an inflammatory biomarker with high specificity and sensitivity to bacterial infection for early diagnosis of VAP and to initiate correct antimicrobial therapy. At present, there are more and more specific studies on VAP at home and abroad. These commonly used indicators are gradually reflected in the value of diagnosis and prognosis of VAP. The study of PCT is one of the hotspots in the field of critical illness.

This clinical study further confirmed that PCT, as a highly specific inflammatory serological marker for bacterial infection, has considerable reliability in the early diagnosis of bacterial infections and compared PCT to other indicators such as CRP and WBC. The specificity and sensitivity of early diagnosis of VAP have great guiding and clinical significance for early diagnosis of VAP.

\section{MATERIALS AND METHODS}

All patients' venous blood was collected on the admission day to detect PCT, WBC and CRP. According to clinical manifestations and bacteriological diagnosis, 66 cases were divided into 2 groups, bacterial infection group. There were positive results of blood culture, sputum and secretion culture in 66 cases of bacterial infection, including 9 cases of chronic obstructive pulmonary disease, 8 cases of asthma, 27 cases of pulmonary infection and 14 cases of bronchitis. There were 5 cases of sepsis, 4 cases of pleural effusion, 4 cases of septic shock and respiratory failure. There were 33 cases of noninfectious group without obvious evidence of bacterial infection, including 10 cases of upper respiratory tract infection, 15 cases of viral pneumonia, 4 cases of bronchial asthma and 4 cases of lung tumor.

One hundred patients with craniocerebral injury requiring mechanical ventilation (MV) for more than $48 \mathrm{~h}$ were divided into the VAP group (50 cases) and nonVAP group (50 cases) according to whether VAP occurred within 5 days after mechanical ventilation. PCT, CRP and WBC were measured and body (T) was recorded before and after mechanical ventilation. The differences of serum PCT, CRP, WBC and T between the 2 groups before and after MV were compared and their sensitivity and specificity to VAP diagnosis were also compared. VAP group was divided into Gram+ve bacteria group and Gram-ve bacteria group according to Gram's staining. The difference of PCT between the 2 groups was compared. The prognosis of VAP group and nonVAP group were compared. All patients with MV were in accordance with the ventilation indications of the guideline for clinical application of MV 2006, deterioration of illness after active treatment; disturbance of consciousness; severe abnormal respiratory patterns, such as respiratory rate $>35-40$ times/min or $<6-8$ times/min, or abnormal respiratory rhythm, or weak or disappearance of spontaneous breathing; blood gas analysis indicated that serious abnormalities were found. Dysfunction of ventilation and/or oxygenation, with $\mathrm{PaO}_{2}<50 \mathrm{mmHg}$, especially after full oxygen therapy $<50 \mathrm{mmHg} ; \mathrm{PaCO}_{2}$ increased progressively, and $\mathrm{PH}$ decreased dynamically. Tracheal intubation was performed by endotracheal intubation, and intubation diameter was selected according to the patient's age and weight. The patients with tracheotomy all accorded with the following indications, expecting or requiring long-term $\mathrm{MV}$; dyspnea due to upper respiratory tract obstruction, such as bilateral vocal 
cord paralysis, neck surgery, neck radiotherapy; repeated aspiration or lower respiratory tract secretion and poor airway clearance; reducing ventilatory dead space; it is helpful for mechanical ventilation support. It is impossible to intubate trachea because of laryngeal stenosis or obstruction.

Preventive tracheotomy is necessary for major head and neck surgery or severe trauma to ensure airway patency. Tracheotomy is more invasive and can cause incision bleeding or infection. Percutaneous dilatation and minimally invasive tracheostomy were performed in tracheotomy. Endotracheal intubation and tracheostomy cannula are equipped with a suction device on the sleeve.

According to the breathing condition, all patients on a respirator should adopt strict preventive measures that are, keeping the airbag pressure of tracheal intubation or incision of tracheal cannula at $25-30 \mathrm{H}_{2} \mathrm{O}$; and strictly abiding by aseptic operation principle when sucking sputum; medical staff should strictly implement hand guard before and after sucking sputum. If there is no contraindication, the head of the bed should be raised by 30 degrees; if there is no contraindication, the patient should be turned over and his back to be patted actively. Oral rinse with $0.2 \%$ chlorhexidine daily, once every $4 \mathrm{~h}$; continuous suction on the capsule; active use of insulin to control blood sugar; respiratory machine thread pipe and humidifier should be replaced 1-2 times a week, when there is obvious secretion change should be timely and more often to prevent contamination, humidifier water should be changed daily and assessment of whether to discontinue the use of sedatives, whether to withdraw and extubate, in order to reduce the number of intubation days.

\section{Specimen treatment:}

The collected venous blood samples were immediately processed in the laboratory. The venous blood samples were centrifuged for $10 \mathrm{~min}$ at $3000 \mathrm{rpm}$ at room temperature. Then the supernatant was aspirated into Eppendorf tubes (EP) and refrigerated at $8^{\circ}$ till analysed. The PCT, CRP and WBC were measured before and after mechanical ventilation and the body temperature was recorded. The changes in the above indices were compared before and after MV and the sensitivity and specificity of each index in the diagnosis of VAP were calculated. PCT levels of the 2 groups were compared. The prognosis of VAP group and nonVAP group were compared.

\section{Determination of serum PCT:}

In this investigation, the electrochemiluminescence assay kit was used for detection of PCT employing the automatic electrochemiluminescence immunoassay system Cobase 411 (Roche). The detection limit was set at $0.01 \mathrm{ng} / \mathrm{ml}$. The double antibody sandwich assay was divided into 4 steps. The first step was to extract 30 $\mathrm{ml}$ samples and monoclonal antibody (in advance). The biotinylated monoclonal PCT antibodies labeled with ruthenium complexes were incubated together to form antigen-antibody complexes; after the antigen-antibody complexes were formed, the magnetic beads coated with streptavidin were added and incubated again, allowing the complexes to bind to the beads above. Biotin is combined with nucleophilic action; The reaction mixture was transferred to the measuring pool, adsorb the magnetic beads on the electrode surface of the measuring pool by the principle of electromagnetic action, and remove the substance which is not combined with the magnetic beads through Pro Cell. The electrodes were added with a certain voltage to make the complex chemiluminescent, and then the luminescence intensity was measured by photomultiplier. Elecsys software was used to calculate the results by the principle of calibration curve.

\section{Determination of serum CRP:}

Using the CRP diagnostic kit and the semi-automatic scattering nephelometer, the basic principle of the determination is the latex immune nephelometry. The reaction sequence is as follows, the serum sample+mouse monoclonal antihuman CRP mixed to form an antigen-antibody complex, which is based on the mouse monoclonal anti CRP. This antibody is allowed to react with the latex and CRP to form an antigenantibody agglutination reaction. The turbidity produced is then determined and the next step of the assay is to determine the CRP concentration in the sample. The specific steps are as follows, the blank/standard/sample mixed with the actual $\mathrm{R}_{1} 100 \mathrm{ml}$ provided by the kit and incubated at $37^{\circ}$ for $5 \mathrm{~min}$ and then mixed with the reagent $\mathrm{R}_{2} 100 \mathrm{ml}$ provided by the kit, incubated for 30 $\mathrm{s}$ in the same environment followed by the absorbance value was read $\mathrm{A}_{1}$; after 4 min, the absorbance was read again to obtain $\mathrm{A}_{2}, \mathrm{~A}=\mathrm{A}_{2}-\mathrm{A}_{1}$; $\mathrm{CRP}(\mathrm{mg} / \mathrm{l}=(\Delta \mathrm{AU} / \Delta \mathrm{AS}) /$ $\mathrm{CS}$, where $\triangle \mathrm{AU}$ is obtained the blank tube absorbance as a control calculation of the sample tube absorbance; $\triangle \mathrm{AS}$ is the blank tube absorbance as a control to obtain the calibration tube absorbance. CS is the CRP concentration measured in the calibration fluid (mg/l). 
WBC counts were obtained using a blood analyzer. The test steps are as follows, $10 \mu \mathrm{l}$ of blood sample is mixed with $2000 \mu \mathrm{l}$ of BA reagent in WBC/BAS cell to form 1:200 diluted solution; It was counted for $6 \mathrm{~s}$ twice under negative pressure $(200 \mathrm{mb}), \mathrm{WBC}$ is counted out and discriminated by boundary markers, providing BAS histogram and the numerical value of WBC, BAS.

\section{PCT detection principle:}

Sandwich immunoassay and final fluorescence detection (ELFA) were used to test the results. Solid phase pipette (SPR) is used as stationary phase and pipetting device. The samples were transferred to the wells of the anticalcitonin antibody (conjugate) labeled with alkaline phosphatase. This procedure allows antigens to bind to immunoglobulins immobilized in the inner walls of SPR, making the conjugates sandwich. Unbound compounds were removed during the washing process. $\mu 1$ There were 2 continuous steps, in each step, the substrate (4-methyl umbelliferone base phosphate) circulates in and out of SPR. A fluorescent product (4-methyl umbelliferone) was prepared by hydrolysis of the substrate catalyzed by the conjugate enzyme and its fluorescence was measured at 450 $\mathrm{nm}$. The fluorescence intensity is proportional to the concentration of antigen in the sample.

Critical patients often have low body resistance, physiological barrier defects, combined with more invasive operations, so easily lead to infection, in addition to the respiratory system, urinary system, abdominal cavity, blood flow, central nervous system and other parts of the infection can occur. In addition, brain injury patients, consciousness disorders are common, cannot effectively collect medical history, symptoms, signs and so on, leading to infection diagnosis difficult, even if the use of PCT such sensitive indicators for infection diagnosis, but cannot determine the location of infection. Therefore, the diagnosis of VAP should first exclude other region infections. In addition to VAP, catheter-related urinary tract infections and intravascular catheter-related infections are the other two major types of infections in ICU, so the emphasis is on their identification.

\section{Statistical analysis:}

SPSS 11 analysis software was used for statistical analysis, and median data and IR (4 quantile interval) were used to measure data. The comparison between the 2 groups was made by 2 independent sample nonparametric tests, and the count data was expressed by the number of cases (percent). The comparison was examined by the four-grid table data. $\mathrm{P}<0.05$ was statistically significant. The data processing methods are as follows. Eqn., $\left\{\Phi(n)=p q-(p+q)+1(p-q)^{2}=\right.$ $(p+q)^{2}-4 p q$, obviously there are: Eqn., $n_{1}(0)=a_{1}(0)$ $\mathrm{n}_{1}(0)+\mathrm{b}_{1}(0) \mathrm{n}_{2}(0) \mathrm{n}_{2}(0)=\mathrm{a}_{2}(0) \mathrm{n}_{1}(0)+\mathrm{b}_{2}(0) \mathrm{n}_{2}(0)$

Adjacency matrix of a simple graph model, Eqn.,

$$
\mathbf{A}=\left[\begin{array}{llllllll}
0 & 1 & 0 & 0 & 0 & 0 & 1 & 0 \\
1 & 0 & 1 & 0 & 0 & 0 & 0 & 0 \\
0 & 1 & 0 & 1 & 0 & 0 & 0 & 1 \\
0 & 0 & 1 & 0 & 1 & 1 & 0 & 1 \\
0 & 0 & 0 & 1 & 0 & 1 & 0 & 0 \\
0 & 0 & 0 & 1 & 1 & 0 & 1 & 0 \\
1 & 0 & 0 & 0 & 0 & 1 & 0 & 1 \\
0 & 0 & 1 & 1 & 0 & 0 & 1 & 0
\end{array}\right]
$$

For a new graph, the following formula was sued to reduce the dimension of the data: Eqn., $\left(\mathrm{U}^{\mathrm{k}}\right) . \varphi(\mathrm{G})=$ $\sum_{\mathrm{i}=1}{ }^{\mathrm{n}} \alpha_{\mathrm{k}}^{\mathrm{i}} \varphi^{\mathrm{T}}\left(\mathrm{G}_{\mathrm{i}}\right) \cdot \varphi(\mathrm{G})=\sum_{\mathrm{i}=1}{ }^{\mathrm{n}} \alpha_{\mathrm{k}}^{\mathrm{i}} \mathrm{k}\left(\mathrm{G}_{\mathrm{i}}, \mathrm{G}\right)$ Its dual form is: Eqn., Min1/2w.w $+\mathrm{C}^{+} \sum_{[\mathrm{i} / \mathrm{yi}=+1]}^{\mathrm{N}+} \xi_{\mathrm{I}}+\mathrm{C}^{-} \sum_{[\mathrm{i} / \mathrm{yi}=-1] \mathrm{I}}^{\mathrm{N}-}$, To resolve the minimization problem, Eqn., $\in_{\text {struct }}(\mathrm{R}, \mathrm{a}, \xi)=$ $\mathrm{R}^{2}+\mathrm{C}_{1} \sum_{1} \xi_{1}+\mathrm{C}_{2} \sum_{\mathrm{i}} \xi_{\mathrm{I}}$,

Trying to solve a constrained quadratic programming problem: Eqn., Min1/2 $\|\omega\|+\mathrm{c} \sum_{\mathrm{i}=1}^{\mathrm{N}}\left(\xi_{\mathrm{i}}+\xi_{\mathrm{i}}^{*}\right)$

$\mathrm{f}_{\mathrm{i}}-\mathrm{y}_{\mathrm{i}} \leq \epsilon+\xi_{\mathrm{i}}$

s.t. $\mathrm{y}_{\mathrm{i}}-\mathrm{f}_{\mathrm{i}} \leq \in+\xi_{\mathrm{i}}^{*}, 1 \leq \mathrm{i} \leq \mathrm{N}$

$\xi_{\mathrm{I},} \xi_{\mathrm{i}}^{*} \geq 0$

The data obtained from the experiment were expressed by means of mean \pm standard deviation, and the measured data were analyzed by paired t test and one-way variance. The comparison among groups was performed using single factor variance analysis of randomized block design. $\mathrm{P}<0.05$ was considered statistically significant.

\section{RESULTS AND DISCUSSION}

Fifty-two patients, 34 males and 18 females, aged 26 to 80 , with an average age of $56.14+10.28$, required MV for more than $48 \mathrm{~h}$. The Glasgow Coma Scale (GCS) was $3-8$ points, with an average of $6.73+2.14$ points. Primary diseases included hypertensive cerebral hemorrhage in 20 cases, cerebral infarction in 12 cases, subarachnoid hemorrhage (SAH) in 11 cases, and meningioma in 8 cases. VAP was divided into VAP group (26 cases) and nonVAP group (26 cases) according to MV for 5 days. There was no significant difference in age, sex, GCS and type of disease between the two groups $(\mathrm{P}>0.05)$, as shown in Table 1. 
The APACHE II scores of the 2 groups were recorded in Table 2 on $\mathrm{d} 1$ and 5 . The scores of the 2 groups were compared in fig. 1 . On $\mathrm{d} 1$, the average score of nonVAP patients was $28.20+5.18$, the average score of VAP patients was $27.16+4.77$, on $\mathrm{d} 5$, the average score of -VAP patients was $15.08+3.72$ and the average score of VAP patients was $16.60+3.16$. Comparing APACHE II scores, the scores of patients on $\mathrm{d} 5$ were lower, but there was no significant difference between VAP group and nonVAP group $(\mathrm{P}>0.05)$.

PCT, CRP, WBC and T were recorded before MV, 48, 72 and $92 \mathrm{~h}$ after MV in VAP group and nonVAP group. There was no significant difference in PCT, CRP, WBC and $\mathrm{T}$ between the 2 groups before MV. Before MV, the PCT value of VAP group was slightly higher than that of nonVAP group. After MV, the PCT value of nonVAP group was slightly higher than that of VAP group. PCT levels were slightly but not significantly elevated $48 \mathrm{~h}$ after MV as shown in fig. 2.

The level of PCT in VAP patients increased slightly. The level of PCT in VAP patients increased linearly from 72 to $96 \mathrm{~h}$ after MV. It can be seen that PCT has significant role in the diagnosis of VAP. Before MV, there was no significant difference in CRP value between the VAP group and nonVAP group. After MV, CRP levels of nonVAP patients increased slightly but not significantly (fig. 3). After MV, the CRP level of patients in VAP group increased slightly at 48, 72 and $96 \mathrm{~h}$ after MV. The level of CRP in VAP patients increased linearly at $24 \mathrm{~h}$, which was consistent with the change of PCT value. CRP significantly assisted diagnosis of VAP.

Before MV, there was no significant difference in WBC value between VAP group and nonVAP group. After MV, WBC level of VAP group and nonVAP group increased, but there was no significant difference between both groups (fig. 4). WBC levels of VAP patients was 48 hours to 96 hours before and after MV. The level of elevation was statistically significant, but the trend was not obvious. Therefore, WBC, as a routine test data, can only be used to diagnose VAP.

There was no significant difference in PCT level between VAP group and nonVAP group before MV. PCT, CRP and WBC increased significantly after VAP (P it has higher sensitivity and specificity. The study also found that the level of PCT in VAP group was significantly higher than that in nonVAP group.

TABLE 1: COMPARISON OF GENERAL INFORMATION BETWEEN TWO GROUPS OF PATIENTS

\begin{tabular}{lccccccc}
\hline Group & Sample & \multicolumn{2}{c}{ Gender } & GCS & \multicolumn{2}{c}{ Type of disease } \\
\hline & & Male & Female & & $\begin{array}{c}\text { Hypertensive } \\
\text { cerebral hemorrhage }\end{array}$ & SAH & $\begin{array}{c}\text { Cerebral } \\
\text { infarction }\end{array}$ \\
VAP & 26 & 16 & 10 & $6.73+1.14$ & 11 & 5 & 6 \\
non-VAP & 26 & 18 & 8 & $6.73+2.91$ & 9 & 6 & 6 \\
\hline
\end{tabular}

TABLE 2: THE COMPARISON OF THE RESULT OF THE TWO GROUPS

\begin{tabular}{|c|c|c|c|c|c|c|}
\hline \multirow{2}{*}{ Variable } & \multicolumn{2}{|c|}{ LNKYJF } & \multicolumn{2}{|c|}{ LNKYRY } & \multicolumn{2}{|c|}{ LNZL } \\
\hline & $\mathrm{HIGH}$ & LOW & $\mathrm{HIGH}$ & LOW & $\mathrm{HIGH}$ & LOW \\
\hline & -3.59 & -3.81 & -3.749 & -3.887 & -6.759 & -6.379 \\
\hline \multirow[t]{2}{*}{ VAP } & $(-15.01)$ & -5.59 & -17.49 & -18.19 & -15.82 & -19.17 \\
\hline & 0.179 & -7.37 & 0.221 & -32.493 & -0.132 & -31.961 \\
\hline \multirow{2}{*}{ CDE } & 2.77 & -9.15 & 1.94 & -46.796 & -1.21 & -44.752 \\
\hline & 0.021 & -10.93 & 0.023 & -61.099 & 0.125 & -57.543 \\
\hline LNER & 0.99 & -12.71 & 2.14 & -75.402 & 2.23 & -70.334 \\
\hline \multirow{3}{*}{ LNPJXS } & -0.078 & -0.014 & 0.031 & 0.041 & 1.182 & 1.162 \\
\hline & -3.08 & -0.48 & 1.78 & 1.67 & 25.14 & 30.71 \\
\hline & -2.59 & 0.008 & 5.0842 & 0.168 & 1.119 & -0.201 \\
\hline \multirow[t]{2}{*}{ CDE $(-1)$} & $(-15.02)$ & 0.23 & 6.633 & 3.22 & 3.995 & -1.29 \\
\hline & -2.25 & -0.022 & 8.18 & 0.044 & 0.2587 & 0.041 \\
\hline LNER(-1) & -2.93 & -0.92 & 9.7324 & 1.97 & 1.746 & 1.71 \\
\hline $\mathrm{ADF}$ & -3.617 & -4.52 & -3.08 & -4.12 & -3.987 & -4.02 \\
\hline Hausman & -4.299 & 8.31 & 8.27 & 8.67 & 7.491 & 8.92 \\
\hline F VALUE & -4.981 & 122.24 & 224.01 & 178.76 & 201.9 & 213.58 \\
\hline $\mathrm{R}_{2}$ & 0.958 & 0.961 & 0.969 & 0.919 & 0.972 & 0.978 \\
\hline TOTAL & 189 & 162 & 189 & 162 & 189 & 162 \\
\hline
\end{tabular}

6 


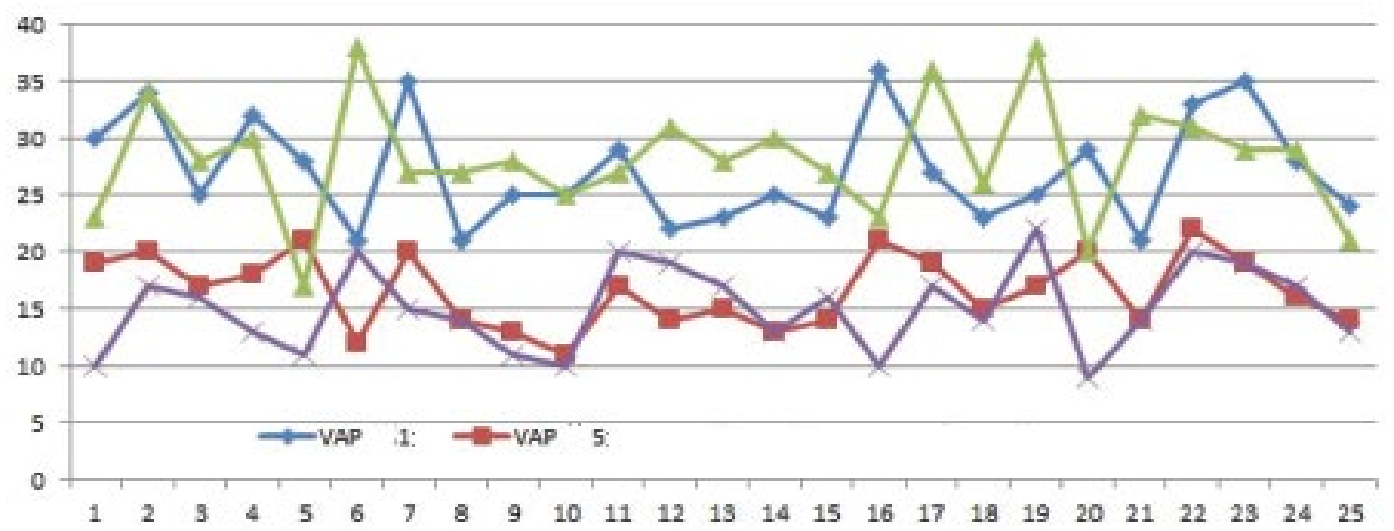

Fig. 1: Comparison of APACHE II Score

\section{$(-\bullet)$ VAP1, (-口-) VAP5}

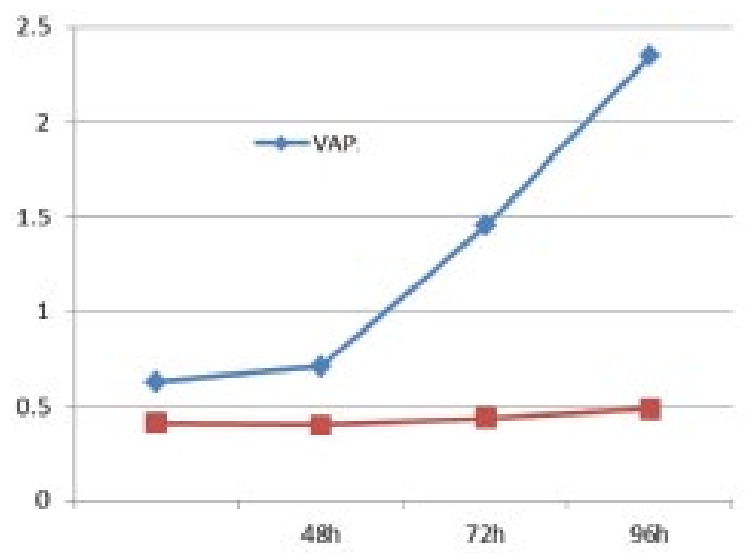

Fig. 2: Comparison of VAP Score $(-\bullet)$ VAP

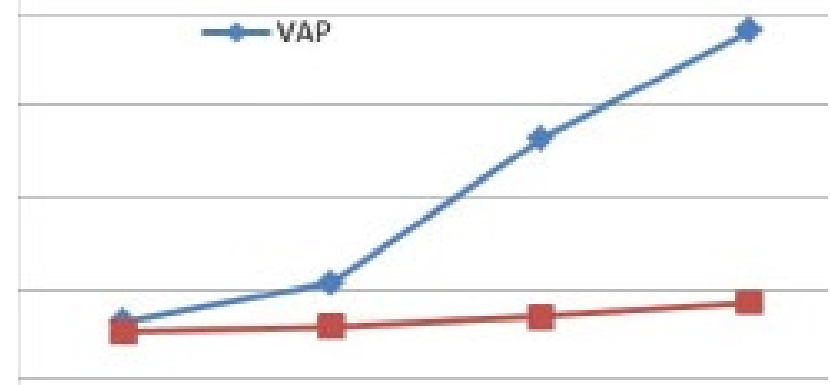

Fig. 3: Comparison of APACHE II score after treatment $(-\bullet)$ VAP

VAP significantly prolonged MV time, ICU time and total hospitalization time, but there was no significant difference in mortality within $1 \mathrm{mo}$, suggesting that VAP may not be an independent cause of death in ICU patients, which is consistent with reported studies.

Previous studies have reported that PCT is a good predictor of VAP outcome and high concentrations of PCT were associated with poor prognosis, but there

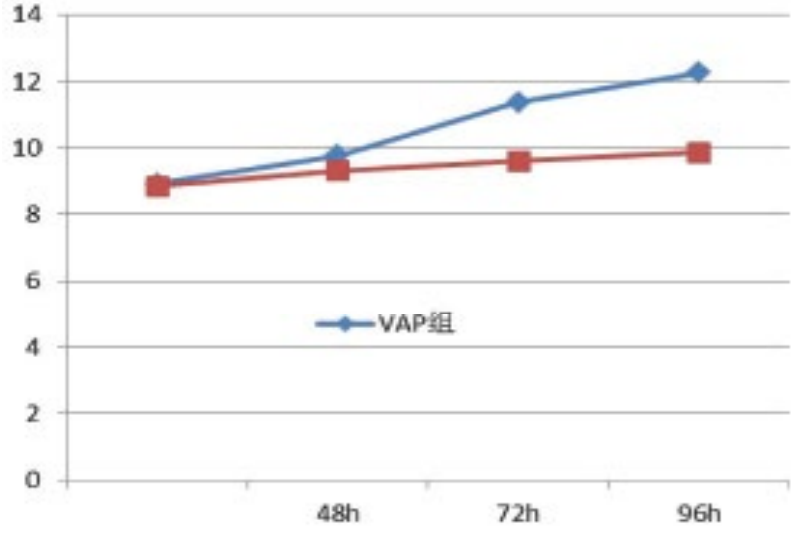

Fig 4: Comparison of VOC score after treatment $(-\diamond-)$ VAP

was no significant correlation between mortality and PCT levels indicating that PCT is not a good predictor of the survival rate of VAP patients.

It has been shown that endotoxins and endotoxins can induce PCT, but there are few and controversial studies on the difference of PCT levels between Gram+ve bacteria and Gram-ve bacteria. This study revealed that the PCT level of Gram-ve bacterial infection was significantluy higher than that of Gram+ve bacterial infection. In a study involving 52 Gram-ve bacterial infections and 45 Gram+ve bacterial infections, which showed that Gram-ve infected parients had higher PCT levels than Gram+ve infected patients and the levels were not affected by the severity of the disease. However, in another study involving 52 patients with systemic inflammatory response syndrome (SIRS), it was found that the plasma levels of IL- 6 , IL-1 $\beta$ and IL18 were elevated in Gram+ve bacterial infection, but CRP, TNF, IL-8, IL-10 and PCT were not elevated.

With the increase of the number of critically ill patients and the prolongation of their life span, there is an increasing trend in ICU infection. PCT is only 
a biological marker for bacterial infections, and it does not distinguish the site of infection. In addition to the ventilator-associated pneumonia discussed in this article, catheter-related bloodstream infections, indwelling catheter-related infections, abdominal infections, soft tissue infections and intracranial infections in the ICU can all lead to serious adverse outcomes.

PCT alone cannot effectively distinguish the source of infection and it is necessary to rely on other clinical manifestations, laboratory tests to further clarify. In addition, PCT has no significant value in the differential diagnosis of VAP caused by fungi or viruses. It can only be determined by changes in lung shadow, sputum, culture results and corresponding laboratory tests. The diagnosis of VAP still requires airway secretions. There have been studies on bacterial volatile organic compounds (VOC) in exhaled gases of patients undergoing mechanical ventilation to determine colonization and infection, as well as specific pathogens.

PCT is a hormone-free glycoprotein, a precursor peptide of calcitonin (CT), consisting of 116 amino acids with a relative molecular weight of $14.5 \mathrm{kDa}$. The encoding gene is located on chromosome 11 . PCT, which is rapidly degraded to 114 amino acids by dipeptidase, appears in the blood circulation and is then degraded into immature CT. It is extremely low in healthy human serum. It is almost impossible to detect, but cytotoxins and cytokines (IL-1 $\beta$, TNF- $\alpha$ ) induce the expression of CTmRNA during inflammation and sepsis and stimulate macrophages and monocytes to secrete large amounts of PCT. This increased expression is manifested in liver, kidney, lung and other tissues, because the parenchymal cells of these tissues lack secretory granules, so unprocessed CT directly released into the blood.

The results of this study of serum PCT in 100 patients with respiratory diseases showed that there was a significant difference in the median of PCT between bacterial infection group and non infection group $(\mathrm{P}<0.01)$. $\mathrm{PCT}$ in bacterial pneumonia was significantly higher than that in viral pneumonia, which may be due to the attenuation of CT mRNA expression by IFN- $\gamma$ produced during viral infection. It is suggested that PCT can be used as an early diagnostic index for bacterial infection and can be used to predict and judge bacterial infection. Therefore, PCT is an important parameter for differential diagnosis of lower respiratory tract infection.
The continuous monitoring of PCT concentration in 5 patients with severe pneumonia showed that PCT level increased significantly, even as high as $60 \mathrm{ng} / \mathrm{ml}$, the higher the PCT level, the more the complications, the higher the mortality. The persistent elevation of PCT indicated poor prognosis and ineffective treatment, while the persistent decrease of PCT indicated that infection is effectively controlled and the condition is improved. In this study, serum PCT $(>0.12 \mathrm{ng} / \mathrm{ml})$ was used as a sensitive marker for bacterial infection of lower respiratory tract.

The sensitivity, specificity, positive predictive value and negative predictive value of serum PCT $(>0.12 \mathrm{ng} /$ $\mathrm{ml}$ ) were higher than those of CRP and WBC. Although the traditional WBC count can indicate the existence of severe bacterial infection, its diagnostic specificity is poor, mainly because the increase in white blood cell count is more suggestive of inflammatory reaction, rather than specific manifestations of infection. CRP is an acute reactive protein, which can be induced by many factors, and the level of serum PCT is not affected by non-infectious factors. Therefore, the diagnostic value of PCT for bacterial infection is significantly higher than WBC count and CRP determination.

It is a new index with high sensitivity and specificity. Because of the unique biodynamic characteristics of PCT, PCT can act as a modulator of inflammatory immune response. The elevation of traditional cytokines in the blood circulation is transient, while PCT increased substantially and persisted in the blood circulation. This specific biodynamic feature provides clinical dynamic detection of PCT levels and timely monitoring of disease progression and outlook. Observation of the efficacy of drugs to provide a theoretical basis, based on the results of PCT testing for treatment, can reduce unnecessary antibiotic use, prevent the emergence of drug-resistant bacteria ${ }^{[6,7]}$. PCT detection is convenient, the detection time is within $20 \mathrm{~min}$, as an early indicator of lower respiratory tract bacterial infection, will play an important role in clinical practice.

\section{REFERENCES}

1. Cohen FS, Fan Z, Patel MS. Classification of rotated and scaled textured images using Gaussian Markov random field models, IEEE Trans. Pattern Anal Machine Intell 1991;13:192-202.

2. Cohen J. Dependency of the spectral reflectance curves of the Munsell color chips. Psychonomic Sci 1964;1:369.

3. Davis LS. Polarograms: A new tool for image texture analysis. Pattern Recognit 1981;13:219-23.

4. Davis LS, Johns S, Aggrawal JK. Texture analysis using generalized co-occurrence matrices, IEEE Trans. Pattern Anal Machine Intell 1979;PAMI-1:251-59. 
5. Funt B, Finlayson G. Color constant color indexing, IEEE Trans. Pattern Anal Machine Intell 1995;17:522-28.

6. Healey G, Jain A. Retrieving multispectral satellite images using physics-based invariant representations, IEEE Trans. Pattern Anal Machine Intell 1996;18:842-48.

7. Siemińska L, Wojciechowska C, Walczak K, Borowski A, Marek B, Nowak M. Associations between metabolic syndrome, serum thyrotropin, and thyroid antibodies status in postmenopausal women, and the role of interleukin-6. EndokrynolPolska 2015;66(5):394-403.

8. Yin J. Relationship between the prevalence of thyroid nodules and metabolic syndrome in the iodine-adequate area of Gangzhou, China: a cross-sectional and cohort study. Int J Endocr 2014;17:675-796.

9. Blusková Z, Koštálová L, Celec P, Vitáriušová E, Pribilincová Z, Maršálková M. Evaluation of lipid and glucose metabolism and cortisol and thyroid hormone levels in obese appropriate for gestational age (aga) born and non-obese small for gestational age (sga) born prepubertalslovak children. J PediatrEndocr Met 2014;27(7-8):693-99.

10. Laterza L, Piscaglia AC, Lecce S, Gasbarrini A, Stefanelli ML. Onset of ulcerative colitis after thyrotoxicosis: A case report and review of the literature. Eur Rev Med PharmacolSci 2016;685-88.

11. Peng XG, Chen ZF, Zhang KJ, Wang PG, Liu ZM, Chen ZJ. Vegftrapon inhibits tumor growth in papillary thyroid carcinoma. Eur Rev Med PharmacolSci 2015;19(2):235-40.

12. Tan L, Wang H, Li C, Pan Y. 16s rdna-based metagenomic analysis of dental plaque and lung bacteria in patients with severe acute exacerbations of chronic obstructive pulmonary disease. J Periodontal Res 2014;49(6):760.

13. Wang S, Song K, Guo X, Hao X, Wang N, Chen J. The association of metabolic syndrome with left ventricular mass and geometry in community-based hypertensive patients among hanchinese. J Res Med Sci 2015;20(10):963.

14. Kowalczyk K, Franik G, Kowalczyk D, Pluta D, Blukacz Ł, Madej P. Thyroid disorders in polycystic ovary syndrome. Eur Rev Med PharmacolSci 2017;21(2):346.
15. Baxi R, Vasan SK, Hansdak S, Samuel P, Jeyaseelan V, Geethanjali FS. Parental determinants of metabolic syndrome among adolescent Asian Indians: A cross-sectional analysis of parent-offspring trios. J Diabetes 2015; 8(4):494-501.

16. Neilands J, Troedsson U, Sjödin T, Davies JR. The effect of delmopinol and fluoride on acid adaptation and acid production in dental plaque biofilms. Arch Oral Biol 2014;59(3):318-23.

17. Bittar DG, Pontes LR, Calvo AF, Novaes TF, Braga MM, Freitas PM. Is the red fluorescence of dental plaque related to its cariogenicity? J Biomed Opt 2014;19(6):65004.

18. Sands KM, Twigg JA, Lewis MA, Wise MP, Marchesi JR, Smith A. Microbial profiling of dental plaque from mechanically ventilated patients. J Med Microbio 2015;65(2):147-59.

19. Takamura T, Ogawa R. Physiological saline injections at acupoints for prevention of muscle cramps in lower extremities caused by hemodialysis. J JpnSoc Pain Clin 2013;11:439-42.

20. Montanari G, Ceschin F, Masotti S, Bravi F, Chinea B, Quartarone G. Observational study on the performance of the narhinel method (nasal aspirator and physiological saline solution) versus physiological saline solution in the prevention of recurrences of viral rhinitis and associated complications of the upper respiratory. Minerva Pediatr. 2010;62(1):17-21.

21. Elboga U, Karaoglan H, Sahin E, Kalender E, Demir HD, Basıbuyuk M. F-18 fdg pet/ct imaging in the diagnostic work-up of thyroid cancer patients with high serum thyroglobulin, negative i-131 whole body scan and suppressed thyrotropin: 8-year experience. Eur Rev Med PharmacolSci 2015;19(3):396-401.

This is an open access article distributed under the terms of the Creative Commons Attribution-NonCommercial-ShareAlike 3.0 License, which allows others to remix, tweak, and build upon the work non-commercially, as long as the author is credited and the new creations are licensed under the identical terms

This article was originally published in a special issue: Special issue on "Animal Models \& Experimental Medicine"

Indian J Pharm Sci 2020:82(1)spl issue4;1-9 